\title{
604.
}

\section{DETERMINATION OF THE ATTRACTION OF AN ELLIPSOIDAL SHELL ON AN EXTERIOR POINT.}

[From the Proceedings of the London Mathematical Society, vol. vi. (1874-1875), pp. 58-67. Read January 14, 1875.]

THE shell in question is the indefinitely thin shell included between two concentric, similar, and similarly situated ellipsoidal surfaces, the density being uniform and the attraction varying as the inverse square of the distance.

It was shown by Poisson that the attraction was in the direction of the axis of the circumscribed cone, and expressible in finite terms; the theorem as to the direction of the attraction was afterwards demonstrated geometrically by Steiner, Crelle, t. XII. (1834), his method being to divide the shell into elements by means of conical surfaces having their vertices at an interior point $Q$; and the investigation was about two years ago completed by Prof. Adams, so as to obtain from it the finite expression for the attraction of the shell. The process was explained in a lecture at which I was present: I did not particularly attend to the details of it; and I now reproduce the solution in my own form, stating, in the first place, the geometrical theorems on which it depends.

\section{Statement of the Geometrical Theorems.}

1. We consider (see figure, p. 305) an ellipsoid, and two corresponding points, an external point $P$, and an internal point $Q$; as will appear, the correspondence is not a reciprocal one. The points are such that each of them is, in regard to the ellipsoid, in the polar plane of the other; moreover $P Q$ is the perpendicular at $P$ to the polar plane of $Q$; that is, $Q$ being regarded as given, then $P$ is determined as the foot of the perpendicular let fall from $Q$ upon its polar plane; to a given position of $Q$ there corresponds thus a single position of $P$. It follows that $P Q$ is the normal at 
$P$ to the confocal ellipsoid through this point; that is, given the position of $P$, then $Q$ is the intersection of the polar plane of $P$ by the normal at $P$ to the confocal ellipsoid. Analytically, to a given position of $P$, there correspond three positions of $Q$, viz. these are the intersections of the polar plane of $P$ by the normals at $P$ to the three confocal surfaces through this point, and the correspondence of the points $P, Q$ is a $(1,3)$ correspondence; but the other two positions of $Q$ are external to the ellipsoid, and we are not concerned with them; we determine $Q$ as above by means of the normal to the confocal ellipsoid.

2. If through the point $Q$ we draw at pleasure a chord $R^{\prime} Q R^{\prime \prime}$, and join the extremities $R^{\prime}, R^{\prime \prime}$ with $P$, then the line $P Q$ bisects the angle $R^{\prime} P R^{\prime \prime}$; whence also $P R^{\prime}: Q R^{\prime}=P R^{\prime \prime}: Q R^{\prime \prime}$, or writing $Q R^{\prime}, Q R^{\prime \prime}=r^{\prime}, r^{\prime \prime}$ and $P R^{\prime}, P R^{\prime \prime}=\rho^{\prime}, \rho^{\prime \prime}$, then $\frac{\rho^{\prime}}{r^{\prime}}=\frac{\rho^{\prime \prime}}{r^{\prime \prime}}$. Putting each of these equal ratios $=\frac{\Omega}{R}$, where $\Omega$ is a length depending on the position of $Q$ but independent of the direction of the chord $R^{\prime} Q R^{\prime \prime}$, then $R$ will be a length depending on the direction of the chord, and if along the chord (say in the sense $Q$ to $R^{\prime}$ ) we measure off from $Q$ a length $Q T,=R$, thence the locus of the extremity $T$ of this line will be an ellipsoid, centre $Q$, similarly situate to the given ellipsoid, say this is the "auxiliary ellipsoid."

Consider now the given ellipsoid and a concentric and similarly situated similar ellipsoid, exterior to and indefinitely near it. To fix the ideas, let the semi-axes of the given ellipsoid be $m f, m g, m h$, and those of the consecutive ellipsoid be $(m+d m) f,(m+d m) g,(m+d m) h$. Producing the chord $R^{\prime} R^{\prime \prime}$ to meet the consecutive ellipsoid in $S^{\prime \prime}, S^{\prime \prime}$, then the radial thicknesses $R^{\prime} S^{\prime}, R^{\prime \prime} S^{\prime \prime}$ of the included shell will be equal to each other, or say each $=\Lambda d m$, where $\Lambda$ is a quantity dependent as well on the position of the point $Q$ as on the direction of the chord $R^{\prime} R^{\prime \prime}$ through this point.

3. Let $2 \phi$ denote the angle $R^{\prime} P R^{\prime \prime}$, or, what is the same thing, let $\phi$ denote either of the equal angles $R^{\prime} P Q, R^{\prime \prime} P Q$; then, $R, \Lambda$ being as above, it is found that $\cos \phi=\frac{m R}{\Lambda}$.

\section{Determination of the Attraction of the Shell.}

4. We may now solve the attraction-problem. We consider the indefinitely thin shell (density unity) included between the given ellipsoid and the consecutive ellipsoid, and attracting the exterior point $P$. We determine the corresponding interior point $Q$, and then dividing the shell into elements by means of indefinitely thin cones having their vertices at $Q$, we consider in conjunction the elements determined by any two opposite cones, say the two opposite cones, having for their axis the chord $R^{\prime} Q R^{\prime \prime}$ and a spherical aperture $=d \omega$. The shell-element at $R^{\prime}$ is

$$
r^{\prime 2} d \omega . R^{\prime} S^{\prime \prime}=r^{\prime 2} \Lambda d \omega d m
$$


its attraction on $P$ is therefore

$$
\frac{r^{\prime 2}}{\rho^{\prime 2}} \Lambda d \omega d m,=\frac{R^{2}}{\Omega^{2}} \Lambda d m d \omega,
$$

and the attractions in the directions $Q R^{\prime}$ and $P Q$ are this quantity multiplied by $\sin \phi$ and $\cos \phi$ respectively.

5. But the shell-element at $R^{\prime \prime}$ exerts upon $P$ the same attraction $\frac{R^{2}}{\Omega^{2}} \Lambda d m d \omega$, and the attractions in the directions $Q R^{\prime \prime}$ and $P Q$ are this quantity multiplied by $\sin \phi$ and $\cos \phi$ respectively: hence the attractions in the directions $Q R^{\prime}, Q R^{\prime \prime}$ exactly counterbalance each other, and there remain only the two equal attractions in the direction $P Q$; viz. this, for either of the elements in question, say for the element at $R^{\prime}$, is

$$
=\frac{R^{2}}{\Omega^{2}} \Lambda \cos \phi d m d \omega
$$

or, substituting for $\cos \phi$ its value, $=\frac{m R}{\Lambda}$, this is

$$
=\frac{m d m}{\Omega^{2}} R^{3} d \omega
$$

Hence the whole attraction of the shell is in the direction $P Q$, its value being

$$
\frac{m d m}{\Omega^{2}} \iint R^{3} d \omega
$$

over the whole solid angle at $Q$; and recollecting that $R$ denotes the radius vector in the auxiliary ellipsoid, we have the volume of this ellipsoid

$$
=\iiint r^{2} d r d \omega=\frac{1}{3} \iint R^{3} d \omega
$$

that is, $\iint R^{3} d \omega=$ thrice the volume of the auxiliary ellipsoid, $=4 \pi F G H$, if $F, G, H$ are the semiaxes of the auxiliary ellipsoid. That is,

$$
\text { Attraction of shell }=\frac{m d m}{\Omega^{2}} 4 \pi F G H .
$$

The problem is now solved; but it remains to prove the geometrical theorems, and to determine the values of the quantities $\Omega, F, G, H$, which enter into the expression for the attraction; and we may also deduce the formula for the attractions of a solid ellipsoid.

\section{Proof of the Geometrical Theorems.}

6. I take

$$
\frac{x^{2}}{f^{2}}+\frac{y^{2}}{g^{2}}+\frac{z^{2}}{h^{2}}=m^{2}
$$

for the equation of the ellipsoid; $a, b, c$ for the coordinates of $P ; \xi, \eta, \zeta$ for those of $Q ; \alpha, \beta, \gamma$ for the cosine-inclinations of the radius $Q R^{\prime}$ to the axes. Hence, in 
the equation of the ellipsoid, substituting for $x, y, z$ the values $\xi+r \alpha, \eta+r \beta, \zeta+r \gamma$, and writing for shortness

$$
\begin{aligned}
& A=\frac{\alpha^{2}}{f^{2}}+\frac{\beta^{2}}{g^{2}}+\frac{\gamma^{2}}{h^{2}} \\
& B=\frac{\alpha \xi}{f^{2}}+\frac{\beta \eta}{g^{2}}+\frac{\gamma \zeta}{h^{2}} \\
& C=\frac{\xi^{2}}{f^{2}}+\frac{\eta^{2}}{g^{2}}+\frac{\zeta^{2}}{h^{2}}-m^{2},(C \text { being therefore negative })
\end{aligned}
$$

we have $r^{\prime},-r^{\prime \prime}$ as the roots of the equation

$$
A r^{2}+2 B r+C=0
$$

viz.

and thence

$$
\frac{2 B}{A}=-r^{\prime}+r^{\prime \prime}, \frac{C}{A}=-r^{\prime} r^{\prime \prime}
$$

$$
r^{\prime}=\frac{-B+\sqrt{B^{2}-A C}}{A}, r^{\prime \prime}=\frac{B+\sqrt{B^{2}-A C}}{A}, r^{\prime}+r^{\prime \prime}=\frac{2 \sqrt{B^{2}-A C}}{A} .
$$

7. Suppose for a moment that the semidiameter parallel to $R^{\prime} R^{\prime \prime}$ is $=m v$; we have evidently $v^{2}=\frac{1}{A}$. And then, if in the central section through $R^{\prime} R^{\prime \prime}$ the conjugate semidiameter is $m u$, the equation of the section referred to these conjugate axes will be $\frac{x^{2}}{m^{2} u^{2}}+\frac{y^{2}}{m^{2} v^{2}}=1$, or say, $y^{2}=m^{2} v^{2}-\frac{v^{2}}{u^{2}} x^{2}$, where $y$ is the coordinate parallel to $R^{\prime} R^{\prime \prime}$, so that, taking the coordinate to belong to the point $R^{\prime}$, we have

$$
y=\frac{1}{2}\left(r^{\prime}+r^{\prime \prime}\right)=\frac{\sqrt{B^{2}-A C}}{A} .
$$

For the exterior surface of the shell, $m$ is to be changed into $m+d m$; hence, $y$ and $m$ alone varying, we have

$$
y d y=m v^{2} d m,=m d m \frac{1}{A},
$$

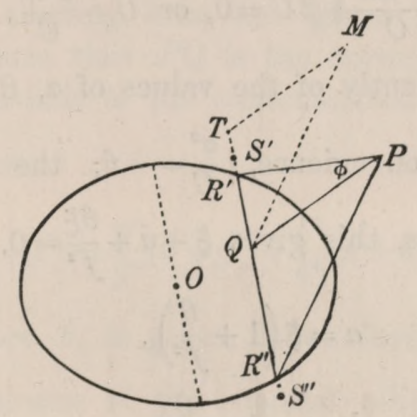

that is,

$$
d y=m d m \frac{1}{\sqrt{B^{2}-A C}}
$$

C. IX. 
viz. this is the value of the radial thickness $R^{\prime} S^{\prime}$ of the shell; or, since the same process applies to the point $R^{\prime \prime}$, we have

$$
R^{\prime} S^{\prime}=R^{\prime \prime} S^{\prime \prime}=m d m \frac{1}{\sqrt{B^{2}-A C}}
$$

or, calling this, as above, $\Lambda d m$, the value of $\Lambda$ is $=\frac{m}{\sqrt{B^{2}-A C}}$.

8. The points $P$ and $Q$ are connected by the condition that, for every direction whatever of the chord $R^{\prime} R^{\prime \prime}$, we have

$$
P R^{\prime}: P R^{\prime \prime}=Q R^{\prime}: Q R^{\prime \prime},
$$

or, what is the same thing, that the line $Q P$ bisects the angle $R^{\prime} P R^{\prime \prime}$. Taking $P R^{\prime}=\rho^{\prime}, P R^{\prime \prime}=\rho^{\prime \prime}$, the condition is $\rho^{\prime}: r^{\prime}=\rho^{\prime \prime}: r^{\prime \prime}$; and taking $(a, b, c)$ as the coordinates of the point $P$, we have

if, for shortness,

$$
\begin{aligned}
\rho^{\prime 2} & =\left(\xi+r^{\prime} \alpha-a\right)^{2}+\left(\eta+r^{\prime} \beta-b\right)^{2}+\left(\zeta+r^{\prime} \gamma-c\right)^{2} \\
& =\sigma^{2}+2 r^{\prime} U+r^{\prime 2}
\end{aligned}
$$

$$
\begin{aligned}
\sigma^{2} & =(\xi-a)^{2}+(\eta-b)^{2}+(\zeta-c)^{2},\left(=\overline{Q P^{2}}\right) \\
U & =\alpha(\xi-a)+\beta(\eta-b)+\gamma(\zeta-c)
\end{aligned}
$$

and similarly

$$
\rho^{\prime \prime 2}=\sigma^{2}-2 r^{\prime \prime} U+r^{\prime \prime 2}
$$

The required condition therefore is

viz. this is

$$
\frac{\sigma^{2}}{r^{\prime 2}}+\frac{2}{r^{\prime}} U=\frac{\sigma^{2}}{r^{\prime \prime 2}}-\frac{2}{r^{\prime \prime}} U
$$

$$
\sigma^{2}\left(\frac{1}{r^{\prime 2}}-\frac{1}{r^{\prime \prime 2}}\right)+2 U\left(\frac{1}{r^{\prime}}+\frac{1}{r^{\prime \prime}}\right)=0
$$

so that, omitting a factor, it becomes

that is,

$$
\sigma^{2}\left(\frac{1}{r^{\prime}}-\frac{1}{r^{\prime \prime}}\right)+2 U=6
$$

$$
\sigma^{2} \cdot \frac{-2 B}{C}+2 U=0, \text { or } U=\frac{\sigma^{2} B}{C}
$$

which must be satisfied independently of the values of $\alpha, \beta, \gamma$.

9. Writing, for greater convenience, $\frac{\sigma^{2}}{\bar{C}}=-\theta$, the equation is $U=-\theta B$, viz. substituting for $U, B$ their values, this gives $\xi-a+\frac{\theta \xi}{f^{2}}=0$, \&c., or say,

$$
\begin{aligned}
& a=\xi\left(1+\frac{\theta}{f^{2}}\right), \\
& b=\eta\left(1+\frac{\theta}{g^{2}}\right) \\
& c=\zeta\left(1+\frac{\theta}{h^{2}}\right)
\end{aligned}
$$


and the assumed relation $\frac{\sigma^{2}}{C}=-\theta$ is

$$
(\xi-a)^{2}+(\eta-b)^{2}+(\zeta-c)^{2}=-\theta\left(\frac{\xi^{2}}{f^{2}}+\frac{\eta^{2}}{g^{2}}+\frac{\zeta^{2}}{h^{2}}-m^{2}\right)
$$

viz. substituting for $a, b, c$ the foregoing values, and omitting a factor $\theta$, this is

$$
\theta\left(\frac{\xi^{2}}{f^{4}}+\frac{\eta^{2}}{g^{4}}+\frac{\zeta^{2}}{h^{4}}\right)=-\left(\frac{\xi^{2}}{f^{2}}+\frac{\eta^{2}}{g^{2}}+\frac{\zeta^{2}}{h^{2}}-m^{2}\right)
$$

or, writing for shortness

the equation is

$$
\frac{1}{\Omega^{2}}=\left(\frac{\xi^{2}}{f^{4}}+\frac{\eta^{2}}{g^{4}}+\frac{\zeta^{2}}{h^{4}}-m^{2}\right),
$$

$$
\theta=-\Omega^{2} C \text {. }
$$

We thus see that, $(\xi, \eta, \zeta)$ being given, $\theta$, and therefore also $(a, b, c)$, are uniquely determined. It may be added that, writing $C=-\frac{\sigma^{2}}{\theta}$, we have $\theta^{2}=\Omega^{2} \sigma^{2}$, or say $\Omega \sigma=\theta$.

10. We have, moreover,

and

$$
\frac{a^{2}}{\theta+f^{2}}=\frac{\xi^{2}}{f^{4}}\left(\theta+f^{2}\right), \& c .
$$

$$
\begin{aligned}
\frac{\xi^{2}}{f^{4}}\left(\theta+f^{2}\right)+\frac{\eta^{2}}{g^{4}}\left(\theta+g^{2}\right)+\frac{\zeta^{2}}{h^{4}}\left(\theta+h^{2}\right) & =\frac{1}{\Omega^{2}} \theta+\frac{\xi^{2}}{f^{2}}+\frac{\eta^{2}}{g^{2}}+\frac{\zeta^{2}}{h^{2}} \\
& =-C+m^{2}+C,=m^{2}
\end{aligned}
$$

whence

$$
\frac{a^{2}}{\theta+f^{2}}+\frac{b^{2}}{\theta+g^{2}}+\frac{c^{2}}{\theta+h^{2}}=m^{2}
$$

or, regarding $(a, b, c)$ as given, $\theta$ is determined as a function of $(a, b, c)$ by this cubic equation; and $\theta$ being (in accordance with the foregoing equation $\theta=-\Omega^{2} C$ ) assumed to be positive, we have $\theta$ the positive root of this equation, and $m^{2}\left(\theta+f^{2}\right), m^{2}\left(\theta+g^{2}\right)$, $m^{2}\left(\theta+h^{2}\right)$ as the squared semiaxes of the confocal ellipsoid through the point $P$. And $\theta$ being known, $\xi, \eta, \zeta$ are, by the foregoing equations $a=\xi\left(1+\frac{\theta}{f^{2}}\right)$, \&c., determined in terms of $\xi, \eta, \zeta$; that is, starting from the given external point $P$, we have the internal point $Q$. And it appears that $P Q$ is the normal at $P$ to the confocal ellipsoid, or, what is the same thing, the axis of the circumscribed cone, vertex $P$.

11. The foregoing equation

$$
\frac{\xi^{2}}{f^{4}}\left(\theta+f^{2}\right)+\frac{\eta^{2}}{g^{4}}\left(\theta+g^{2}\right)+\frac{\zeta^{2}}{h^{4}}\left(\theta+h^{2}\right)=m^{2}
$$

considering $a, b, c$, and therefore $\theta$, as given, shows further that the point $Q$ is situate on an ellipsoid which is the inverse of the confocal ellipsoid $\frac{x^{2}}{\theta+f^{2}}+\frac{y^{2}}{\theta+g^{2}}+\frac{z^{2}}{\theta+h^{2}}=m^{2}$ in regard to the given ellipsoid $\frac{x^{2}}{f^{2}}+\frac{y^{2}}{g^{2}}+\frac{z^{2}}{h^{2}}=m^{2}$. 
12. Expressing $\Omega$ in terms of $a, b, c$, we have

$$
\frac{1}{\Omega^{2}}=\frac{a^{2}}{\left(\theta+f^{2}\right)^{2}}+\frac{b^{2}}{\left(\theta+g^{2}\right)^{2}}+\frac{c^{2}}{\left(\theta+h^{2}\right)^{2}} .
$$

We have $\sigma^{2}=\frac{\theta^{2}}{\Omega^{2}},=C^{2} \Omega^{2}$, and

whence

$$
\begin{aligned}
U & =\alpha(\xi-a)+\beta(\eta-b)+\gamma(\zeta-c), \\
& =-\theta\left(\frac{\alpha \xi}{f^{2}}+\frac{\beta \eta}{g^{2}}+\frac{\gamma \zeta}{h^{2}}\right), \quad=-\theta B, \quad=B C \Omega^{2}
\end{aligned}
$$

or, since

$$
\begin{aligned}
\frac{\rho^{\prime 2}}{r^{\prime 2}}=\frac{\sigma^{2}}{r^{\prime 2}}+2 U \frac{1}{r^{\prime}}+1, & =\frac{C^{2} \Omega^{2}}{r^{\prime 2}}+\frac{2 B C \Omega^{2}}{r^{\prime}}+1, \\
& =C \Omega^{2}\left(\frac{C}{r^{\prime 2}}+\frac{2 B}{r^{\prime}}\right)+1 ;
\end{aligned}
$$

this is

$$
A+2 B \frac{1}{r^{\prime}}+C \frac{1}{r^{\prime 2}}=0
$$

$$
\begin{aligned}
\frac{\rho^{\prime 2}}{r^{\prime 2}} & =-A C \Omega^{2}+1=\Omega^{2}\left(\frac{1}{\Omega^{2}}-A C\right) \\
& =\frac{\Omega^{2}}{R^{2}}, \text { if } \frac{1}{R^{2}}=\frac{1}{\Omega^{2}}-A C .
\end{aligned}
$$

This last equation may also be written

$$
\frac{1}{R^{2}}=\frac{1}{\Omega^{2}}\left(\alpha^{2}+\beta^{2}+\gamma^{2}\right)-C\left(\frac{\alpha^{2}}{f^{2}}+\frac{\beta^{2}}{g^{2}}+\frac{\gamma^{2}}{h^{2}}\right)
$$

or, what is the same thing,

$$
\frac{1}{R^{2}}=\frac{\alpha^{2}}{F^{2}}+\frac{\beta^{2}}{G^{2}}+\frac{\gamma^{2}}{H^{2}}
$$

if for shortness

$$
\begin{aligned}
& \frac{1}{F^{2}}=\frac{1}{\Omega^{2}}-\frac{C}{f^{2}}, \\
& \frac{1}{G^{2}}=\frac{1}{\Omega^{2}}-\frac{C}{g^{2}}, \\
& \frac{1}{H^{2}}=\frac{1}{\Omega^{2}}-\frac{C}{h^{2}}
\end{aligned}
$$

viz. substituting herein for $C$ its value $-\frac{\theta}{\Omega^{2}}$, these equations give

$$
F=\frac{\Omega f}{\sqrt{\theta+f^{2}}}, \quad G=\frac{\Omega g}{\sqrt{\theta+g^{2}}}, \quad H=\frac{\Omega h}{\sqrt{\theta+h^{2}}} ;
$$

where $\Omega$ stands for its expression in terms of $a, b, c$. 
13. The expression for $\frac{1}{R^{2}}$ shows that $R$ is the radius vector, cosine-inclinations $\alpha, \beta, \gamma$, in an ellipsoid semi-axes $F, G, H$, which may be regarded as having its centre at $Q$; viz. this is the "auxiliary ellipsoid." And this being so, we have

$$
\frac{\rho^{\prime}}{r^{\prime}}=\frac{\rho^{\prime \prime}}{r^{\prime \prime}}=\frac{\Omega}{R}
$$

It appears from these equations that, drawing from $Q$ parallel to $P R^{\prime \prime}$ a line $Q M,=\Omega$, and from its extremity $M$ parallel to $P Q$ a line to meet $Q R^{\prime}$ in $T$, the locus of $T$ is the auxiliary ellipsoid.

14. By what precedes, the angles $R^{\prime} P Q, R^{\prime \prime} P Q$ are equal to each other, say each is $=\phi$; the triangle $R^{\prime} P R^{\prime \prime}$ gives

that is,

$$
\cos 2 \phi=\frac{\rho^{\prime 2}+\rho^{\prime \prime 2}-\left(r^{\prime}+r^{\prime \prime}\right)^{2}}{2 \rho^{\prime} \rho^{\prime \prime}}
$$

$$
\cos ^{2} \phi=\frac{\left(\rho^{\prime}+\rho^{\prime \prime}\right)^{2}-\left(r^{\prime}+r^{\prime \prime}\right)^{2}}{4 \rho^{\prime} \rho^{\prime \prime}}
$$

viz. this is

$$
\begin{aligned}
& =\left(\frac{\Omega^{2}}{R^{2}}-1\right)\left(r^{\prime}+r^{\prime \prime}\right)^{2} \div 4 \frac{\Omega^{2}}{R^{2}} r^{\prime} r^{\prime \prime}, \\
& =R^{2}\left(\frac{1}{R^{2}}-\frac{1}{\Omega^{2}}\right) \frac{\left(r^{\prime}+r^{\prime \prime}\right)^{2}}{4 r^{\prime} r^{\prime \prime}} \\
& =-A C R^{2} \cdot \frac{4\left(B^{2}-A C\right)}{A^{2}} \cdot \frac{-A}{4 C} \\
& =R^{2}\left(B^{2}-A C\right)
\end{aligned}
$$

or say

$$
\cos \phi=R \sqrt{B^{2}-A C} ;
$$

a remarkable equation which may also be written

$$
\cos \phi=\frac{R}{v^{2}} \cdot \frac{1}{2}\left(r^{\prime}+r^{\prime \prime}\right)
$$

if, as before, $v$ is the semi-diameter parallel to $R^{\prime} R^{\prime \prime}$.

In virtue of the equation $\Lambda=\frac{m}{\sqrt{B^{2}-A C}}$ which defines $\Lambda$, the equation becomes

$$
\cos \phi=\frac{m R}{\Lambda}
$$

and we thus complete the demonstration of the several geometrical theorems upon which the investigation was founded. 
Analytical Expressions for the Attraction of the Shell, and for the Resolved Attractions.

15. The attraction of the shell was shown to be

$$
=\frac{m d m}{\Omega^{2}} 4 \pi F G H ;
$$

or, since the mass of the shell, the density being unity, is

$$
\frac{4 \pi}{3} f g h .3 m^{2} d m=4 m^{2} d m \pi f g h,
$$

we have

$$
\text { Attraction } \div \text { Mass }=\frac{1}{m \Omega^{2}} \frac{F G H}{f g h}
$$

which, by what precedes, is

$$
=\frac{\Omega}{m \sqrt{\left(f^{2}+\theta\right)\left(g^{2}+\theta\right)\left(h^{2}+\theta\right)}}
$$

where

$$
\frac{1}{\Omega^{2}}=\frac{a^{2}}{\left(f^{2}+\theta\right)^{2}}+\frac{b^{2}}{\left(g^{2}+\theta\right)^{2}}+\frac{c^{2}}{\left(h^{2}+\theta\right)^{2}},
$$

$\theta$ being the positive root of

$$
\frac{a^{2}}{f^{2}+\theta}+\frac{b^{2}}{g^{2}+\theta}+\frac{c^{2}}{h^{2}+\theta}=m^{2}
$$

16. It is to be observed that the cosine-inclinations of the line $P Q$ to the axes are

$$
\frac{a \Omega}{f^{2}+\theta}, \frac{b \Omega}{g^{2}+\theta}, \frac{c \Omega}{h^{2}+\theta},
$$

respectively; so that, considering, for instance, the attraction parallel to the axis of $x$, we have

$$
\text { Resolved Attraction } \div \text { Mass }=\frac{a \Omega^{2}}{m\left(f^{2}+\theta\right) \sqrt{\left(f^{2}+\theta\right)\left(g^{2}+\theta\right)\left(h^{2}+\theta\right)}} .
$$

$$
\text { Resolved Attractions of the Ellipsoid } \frac{x^{2}}{f^{2}}+\frac{y^{2}}{g^{2}}+\frac{z^{2}}{h^{2}}=1 \text {. }
$$

17. We may find the attraction of the solid ellipsoid

$$
\frac{x^{2}}{f^{2}}+\frac{y^{2}}{g^{2}}+\frac{z^{2}}{h^{2}}=1
$$

For this purpose, dividing it into shells, semi-axes $m f, m g, m h$, and $(m+d m) f,(m+d m) g$, $(m+d m) h$ respectively, we have for the shell in question

$$
\text { Resolved Attraction } \div \text { Mass }=\frac{a \Omega^{2}}{m\left(f^{2}+\theta\right) \sqrt{\left(f^{2}+\theta\right)\left(g^{2}+\theta\right)\left(h^{2}+\theta\right)}}
$$


and the mass of the shell is $\frac{4 \pi}{3} f g h .3 m^{2} d m$, where the first factor is the mass of the ellipsoid; whence

$$
\text { Resolved Attraction } \div \text { Mass of Ellipsoid }=\frac{a \cdot 3 m \Omega^{2} d m}{\left(f^{2}+\theta\right) \sqrt{\left(f^{2}+\theta\right)\left(g^{2}+\theta\right)\left(h^{2}+\theta\right)}}
$$

$\theta$ being here a function of $m$, and $m$ extending from 0 to 1 . But taking $\theta$ as the variable in place of $m$, the equation

gives

$$
\frac{a^{2}}{f^{2}+\theta}+\frac{b^{2}}{g^{2}+\theta}+\frac{c^{2}}{h^{2}+\theta}=m^{2}
$$

$$
-\frac{1}{\Omega^{2}} d \theta=2 m d m ; \text { that is, } 3 m \Omega^{2} d m=-\frac{3}{2} d \theta .
$$

Moreover $m=0$ gives $\theta=\infty$, and $m=1$ gives $\theta=i$ its value as defined by the equation

$$
\frac{a^{2}}{f^{2}+\theta}+\frac{b^{2}}{g^{2}+\theta}+\frac{c^{2}}{h^{2}+\theta}=1
$$

so that, reversing the sign, the limits are $\infty, \theta$; or, finally, writing under the integral sign $\phi$ in place of $\theta$, the formula is

Resolved Attraction $\div$ Mass of Ellipsoid $=\frac{3}{2} a \int_{\theta}^{\infty} \frac{d \phi}{\left(f^{2}+\phi\right) \sqrt{\left(f^{2}+\phi\right)\left(g^{2}+\phi\right)\left(h^{2}+\phi\right)}}$ which is a known formula. 